\title{
Determinism and the Mystery of the Missing Physics $^{1}$ \\ Mark Wilson, University of Pittsburgh
}

In practice, "classical mechanics" resembles a stool constructed of six or seven legs of unequal lengths: if we unwisely place too much weight in the wrong place while it perches primarily upon legs 1,3 , and 5, it's liable to rock over to legs 2, 4 and 5 in response, perhaps depositing us abruptly on the floor in the process. Throughout our educations we have been encouraged to speak of "classical mechanics" as if it represented a unitary and well understood doctrine. 'Tain't so, but, for many purposes, this little fiction creates no difficulties because the speaker may have simply intended to draw a mathematical contrast whose content is clear from the context, as usually occurs when, e.g., one compares "quantum and classical statistical mechanics." But for many of the questions that philosophers pose, such easy disambiguation is not

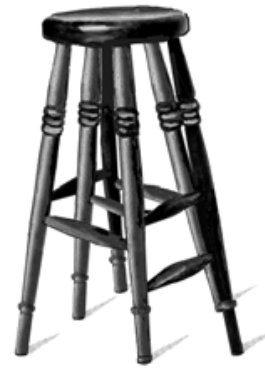
available and such inquiries can become seriously confused unless the wobbly architecture that lies behind the placid facade of "classical mechanics" is adequately recognized. Contemporary commentary on philosophical theories of matter of the eighteenth and nineteenth centuries is often greatly compromised, for example, by its inclination to utilize terminology like "classical mechanics," "the Newtonian picture," etc. as if these phrases represent precise surgical instruments, when they actually conceal multiple blades that cut in incompatible directions.

Philosophers of science often select some specific mathematical formalism (sometimes only implicitly) to represent "classical physics" in their argumentation when that formalism is often incapable of bearing the doctrinal weight expected of it. As a common case in point, many writers begin their investigations by blithely stating "I here adopt Lagrangian formalism as a suitable embodiment of Newtonian theory" without apparently recognizing the serious descriptive holes to which that formalism is heir. Indeed, I can freely confess to have fallen amongst this cohort in my callow years (indeed, I began to study "classical mechanics" more critically because I dimly recognized that had to be something fishy in what I had claimed). In point of fact, classical Lagrangian mechanics, under its normal interpretation, tolerates especially gaping holes in its scope of coverage and, in applied engineering practice, one is commonly forced to say, "Oh, those tools simply won't work in this case; you need to utilize formalism X instead" (I'll supply some examples later). A failure to recognize these descriptive gaps often vitiates the plausibility of whatever lesson it was that our unwitting author had hoped to 
extract.

Unfortunately, --and this is the chief moral of the essay--, we're unlikely to find any wholly stable X upon which "classical mechanics" can permanently and happily rest. The reason for this is simple: classical mechanics represents a set of doctrines admirably suited to macroscopic experience and, in consequence of this large scale mission, must inevitably compress swatches of very complicated physical behavior into simplified rules of thumb. In this essay we shall be especially concerned with some of the popular recipes that classical modelers utilize to conveniently patch over the complicated manners in which extended solids contact one another. Much of the multi-leggedness we witness in the classical stool traces to the fact that such "rules of thumb" for contact action exist in a variety of flavors that are not compatible with one another from a foundational point of view, yet possess equal bragging rights to qualify as "the classical approach" to the contact between solids.

To demonstrate how dramatically show how dramatically such seemingly technical issues affect matters of philosophical concern, let us examine how the question "is Newtonian mechanics deterministic?" turns upon these concerns. Recently John Norton ${ }^{2}$ has describes a situation involving a ball sliding down a peculiarly shaped dome which looks, at first appearance, as if it must prove indeterministic from a classical point of view. However, the conceptual situation is not as straightforward as first appears, for foundational multi-leggedness enters the discussion in a hidden yet quite significant way. Accordingly, an examination of Norton's example can serve as an excellent illustration of the unstable gappiness that represents the natural price classical mechanics must pay to achieve the phenomenal successes it achieves on the macroscopic level (this is a chief theme in my recent book $^{3}$; in this paper I'll be able to identity the gaps, but not explain their virtues).

Norton's example represents an improved recasting of a sort of example that has been long familiar in the physical literature. He allows a ball of unit mass to slide frictionlessly under terrestrial gravitation down a concave hillside track with the resultant equation of motion $\mathrm{d}^{2} \mathrm{r} / \mathrm{dt}^{2}=\mathrm{r}^{1 / 2}$, where $\mathrm{r}$ is arc length along the track. The lack of a Lipshitz bound upon the active force at the apex creates a situation where this equation tolerates many possible solutions for a ball situated at the apex with no initial velocity (it can stay put or slowly start to roll down the track $\underline{a b}$ libitum with $\left.r(t)=1 / 144 t^{4}\right)$. The curvature of the track "turns off" the active part of the gravitational force acting on the ball as the origin is approached, yet turns it back on very rapidly as the ball is displaced from the apex, allowing our moving 
ball solutions to decelerate smoothly enough to display a limiting zero velocity as the origin is approached. Given such a counterexample (many others can be easily constructed), why has Newtonian mechanics heretofore so often been described as "deterministic"? Norton presumes that he has shown that this familiar characterization is simply wrong, but, in fact, sundry "six-legged stool" considerations concerning the phrase "Newtonian mechanics" affect this example in substantive ways, as we shall establish in the course of this review. For such reasons, Norton's case nicely illustrates the considerable care we must observe we ponder the "content" of "Newtonian mechanics."

To this end, we should distinguish three basic families of foundational approach to "classical mechanics" (they split into further subdivisions as further questions are pressed): (MP) mass point particle mechanics (the "classical physics" usually taught to freshman physics majors), (PC) the physics of rigid bodies and perfect constraints (commonly introduced as "analytical mechanics" in that same freshman course in a somewhat sureptitious manner) and (CM) continuum mechanics (taught systematically only to theoretical engineers and applied mathematicians nowadays). The "objects" basic to these formulations are, respectively, (MP) unextended point-masses, (PC) extended yet perfectly rigid geometrical shapes such as balls and curved tracks and (CM) shapes that are thoroughly flexible at every size scale, such as distorting balls, pliable tracks, wooden beams and fluids. Probably class $(\mathrm{CM})$ represents the most natural embodiment of the doctrines we traditionally expect to find in "classical mechanics" but articulating its operative mathematics precisely is rather tricky and often reserved for specialists. For this reason, point mass-like formulations appear to be "foundational" within historical textbooks such as Thompson and Tait's Treatise on Natural Philosophy simply because a proper framework for articulating continuum mechanics principle directly lay beyond their technical reach. But their intended "world view" was one of continua, not point masses. In any case, our three basic approaches are not, from a foundational point of view, strictly compatible with one another: the modes of description favored as basic in one approach can only be treated as convenient approximations within the others. Into which of these foundational categories does Norton's case fall?

His ball (really, a point mass) and track comprise objects of a fixed geometry and hence represent a standard arrangement within (PC) the physics of perfect 
constraints. This approach typically makes two characteristic assumptions about cases like Norton's. (a) A free particle swooshing along the constrained surface will follow a geodesic of the surface with a constant speed relative to the surface (this venerable doctrine is commonly called "generalized inertia"). ${ }^{4}$

(b) Any total force exerted against the system can be perfectly decomposed into two disjoint components: the part that attempts to drive the particle unsuccessfully into the surface and the piece able to affect its generalized inertial motion. Forces in the first group are commonly dubbed "constraint forces," "forces of reaction" or "forces that do no work" and the latter, "active forces" or "forces that perform work." In Norton's example, the total applied force appears as the constant downward directed gravitational force, which is then apportioned into my two sub-categories in different amounts at different positions along his dome. At the very top the gravitational force proves

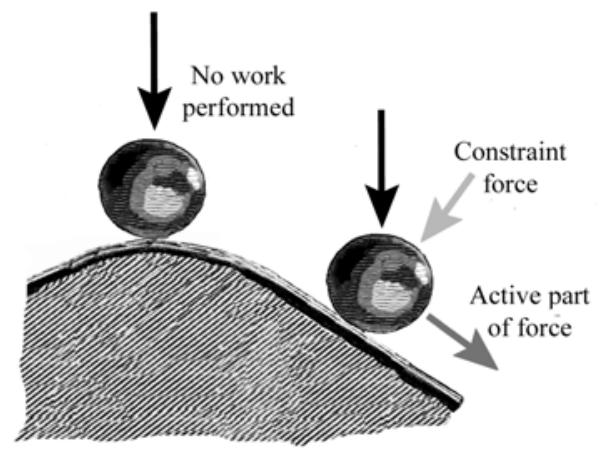
completely ineffectual in its capacity "to perform work": it entirely operates entirely as a "constraint force" there. However, as the particle moves away from the summit, some portion of the impressed gravitational force begins to "turn on" as an active force. Apportioning "forces" into these bifurcated categories is a very ancient practice--it is implicit in Greek thinking about statics--and an average textbook evokes the distinction without a murmur of apology (this "sneakiness" allows analytical mechanics to creep on stage).

Nonetheless, from the points of view of our alternative foundational starting points, this kind of "active/reactive" decomposition is strictly unwarranted and can only be justified as a form of convenient approximation. Any friend of determinism should be cautious about allowing forces to be glibly divided into "reactive" and "active" categories, for that's how Norton's loss of determinacy secretly enters the scene.

In orthodox mass point mechanics (MP), by contrast, its unextended particles obey strict First Law inertia: unaffected particles travel in straight lines without change of velocity. Moreover, Newton's Third Law, as it is commonly understood in a MP context, rules out the possibility of true "constraint forces" entirely: it can't tolerate forces that strictly bind a particle to a fixed geometrical constraint. At best, the particle will stay pretty near a geodesic on the constraining surface while simultaneously displaying a lot of rapid wiggling. It can then prove convenient to factor away the wiggling as a "fast motion" that is superimposed 
upon a "slow motion" in the manner described in every textbook on approximation theory, but this decomposition is to be tolerated only as an approximation. In other words, orthodox MP mechanics rejects the assumption that forces can be strictly apportioned into "active" and "constraint" classes: all forces are wholly "active."

To see this, observe that a standard textbook treatment of Newton's Third Law replaces its vague original "action = reaction" formulation by three tacit assumptions: that fundamental forces always arise in inter-particle pairs that are (1) central, (2) balanced and (3) dependent only upon relative positions, not velocities. These assumptions then allow the textbook to associate any particle system with a potential energy function and to prove the conservation of energy on that basis. Within rival foundational stories such as $\mathrm{CM}$, the conservation of energy is often adopted as an independent postulate, but the Third Law derivation just sketched represents the usual way its status is approached within freshman textbooks and in the more rigorous developments based squarely upon the MP picture.

But let's now scrutinize the "constraint force" that binds our particle a to Norton's track from the MP point of view. According to First Law inertia, $\underline{\text { a }}$ should normally wish to whoosh ahead along a tangent running off the surface but we have also assumed that the track's matter supplies just enough "constraint force" $f_{a}$ to pull a back to the surface with exactly the right velocity to satisfy the expectations of generalized inertia. Okay, but let us now run a particle $\underline{b}$ along the track that is exactly like a except that it scoots a little faster along the path. Once again the track's "constraint force" must exert a binding force $f_{b}$ of the right magnitude to pull $\underline{\mathrm{b}}$ down to its appointed, generalized inertia rendezvous with the hillside. ${ }^{5} \mathrm{f}_{\mathrm{b}}$ clearly needs to be different from $\mathrm{f}_{\mathrm{a}}$. But how can the constraining surface prove smart enough to exert the exact degree of force required? Answer: it must be able to "see" the velocity difference between $\underline{\mathrm{a}}$ and $\underline{\mathrm{b}}$ and adjust its strength accordingly. But

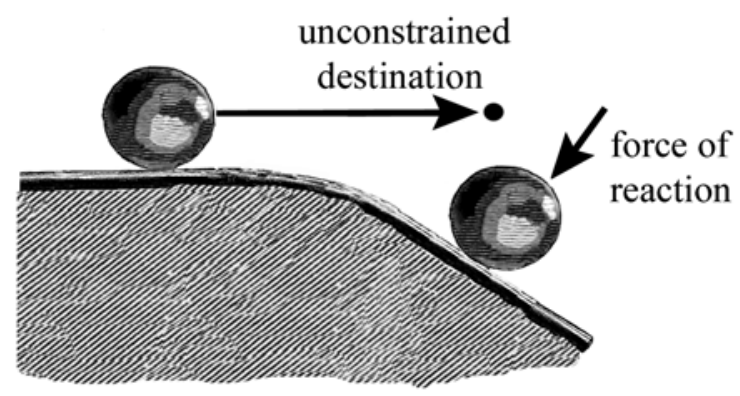
permitting such a sensitivity to velocity is contrary to the Third Law and the canonical "conservation of energy" story. In fact, the MP foundational framework can legitimately tolerate strong binding forces that can only approximately drag $\underline{\mathrm{a}}$ and $\underline{\mathrm{b}}$ along similar "slow motion" paths, with residual differences showing up in the different "fast motion" ways in which $\underline{\text { a }}$ and $\underline{b}$ wiggle upon the hillside. Strictly speaking, the MP particle picture doesn't 
tolerate true "forces of constraint." Indeed, with respect to their fundamental qualities, "active forces" and "constraint forces" should be regarded as incompatible sorts of conceptual critter, a tension that rumbles underneath many of the great historical disputes about the nature of "force." 6

It is rather surprising that these elementary issues are rarely discussed clearly. A notable exception can be found in Giovanni Gallavotti's excellent Elements of Mechanics, where he seems flabbergasted by the manner in which most textbooks sneak analytical mechanics on stage through nothing more than inadequate "forces that do or don't do work" hand-waving:

However, the principle of the conservation of the difficulties makes it clear that there must be some serious obstacle to the actual applications of such a shining but simplistic vision. The true constraints are, in fact, generated by forces that...generally are neither simple nor conservative...but depend upon the velocities of the points as well as their positions. ${ }^{7}$ He credits V.I. Arnold with first recognizing that appeals to constraints should be properly approached within the framework of (MP) as a kind of approximation theorem involving rapidly jittery motions superimposed upon a slower trend.

The only significant comment I would add to Gallavotti's discussion is that we should remain aware of the fact that the "physics of perfect constraints" (PC) can also be approached profitably from the continuum physics side of things (CM), where the alternative tale of approximation we will then weave will look significantly different from the MP story just sketched. In terms of predictive realism, the $\mathrm{CM}$ version of the story is preferable because it provides better warnings of the many circumstances where "analytical mechanics" $(=\mathrm{PC})$ winds up treating real life tracks, domes and balls quite poorly. ${ }^{8}$ We shall come back to the CM side of the ledger later.

What, then, should we say about the "determinism" of MP particle physics? Here we confront the basic phenomena of "missing physics" that represents my main theme in this essay. One can't immediately produce a definitive answer to the "determinism" question simply because standard presentations invariably weasel quite a bit with respect to foundational assumptions that must be settled before a feature such as determinism can be coherently adjudicated. In particular, we must know more than we are usually told about the exact kinds of inter-particle forces that our MP physics will tolerate. To be sure, our textbook will tell us about universal gravitation and Coulomb's law, but what about the strong local forces responsible for molecular binding and repulsion? In real life practice, molecular modelers evoke various inverse sixth and twelfth power laws (such as the wellknown Lennard-Jones potential) for these purposes, but they are usually rather 
evasive about the classical validity of these "rules of thumb." Instead, they wax weasely: "I don't really know any classical contender for a short range force law that should be accepted as a canonical part of MP physics, but my simple power law rules work pretty well as a stopgap." Here we witness a typical "missing physics" gap within point mass mechanics where practitioners commonly evoke fairly coarse rules of thumb to fill foundational gaps that they are happy to abandon if things don't work out right.

Yet as long as such gaps remain unfilled, it becomes misleading to classify point particle physics as "deterministic" or not--the question simply can't be adjudicated on a rational basis with such incomplete information. In the breech, we will do better as philosophers, I think, to concentrate directly upon the descriptive holes presently tolerated within assembled MP doctrine, rather than focusing upon indeterminism per se. In 1962 Richard Montague ${ }^{10}$ carefully formalized the treatment of "point particle physics" that had been provided by Patrick Suppes and his groups in hopes of rigorously establishing traits like determinism and found, to his dismay, that his collected materials were far too feeble to address this question in a remotely interesting fashion. Indeed, Suppes et al had been so timid in their formulations that they had omitted the Third Law altogether! ${ }^{11}$ In such a context, asking "Is classical physics deterministic?" resembles "Have you stopped beating your wife?": vital background presumptions need to directly addressed first.

However, if we are willing to follow current modeling practice and strengthen our Third Law principles sufficiently to further demand that all interparticle interactions must obey some kind of power law principle, then we can guarantee that only analytic functions (away from the collision singularities) will appear in our governing ordinary differential equations. If so, then Cauchy's original local existence proof based upon power series expansions kicks in where the uniqueness of trajectories comes along for free (we only need worry about Lipschitz conditions and all that when some lapse of smoothness intrudes upon our starting set of equations). Under this supplementary assumption, MP physics becomes provably deterministic.

Which is not to say that point mass physics therefore left in descriptively great shape, because it is notorious that its local trajectories are not always globally extendible: its particles can either bump into one another or allow some required quantity (such as position) to blow up into an intolerable infinity within finite time. ${ }^{12}$ Such unpleasant behaviors failures of "global existence" have prompted classical physicists to invent all sorts of excuse for papering over these descriptive 
holes within their doctrines, a point to which I'll soon return. But it is as descriptive holes such issues should be addressed, not as failures of determinism: perfectly harmless-looking point particle setups often engender descriptive impossibilities after a time when left to their own devices.

I stress the advisability of looking at matters in this manner because John Earman in his well-known book on determinism ${ }^{13}$ (and John Norton follows him in this) artificially converts a classic "blow up" phenomenon into an alleged "failure of determinism" through recasting the proper meaning of "initial condition" in a manner that I regard as both terminologically unfortunate and contrary to well established mathematical practice. ${ }^{14}$ The only motive I can discern for this reclassification is that it allows one to inform other philosophers that "I have discovered that classical particle mechanics isn't really deterministic" rather than the more apt "I have discovered that classical particle mechanics sometimes tolerates strange descriptive gaps." The former asseveration may sound more thrilling, but I think the latter statement better captures the circumstances to which we should pay closer attention as philosophers of science.

When asked about the collision and "blow up" problems characteristic of point particle mechanics, physicists commonly reply in one of two ways: (i) they claim that some unspecified "missing physics" will kick in to prevent the blowup from occurring or (ii) they'll suggest some relatively crude rule of thumb to "continue" trajectories past their apparent breakdown calamities. As an example of the first reply, it is often suggested that the celebrated Xia blow up in point particle gravitation will be "cured" in real life by the repulsive mechanisms that supply planets with their real life "size," for such barriers should prevent the system from milking the infinite potential wells tolerated within the unadulterated Xia set up. But, as we noted with the molecular modelers, the exact nature of these "repulsive mechanisms" is left unspecified in canonical MP physics. A good example of the second response is the rule that colliding particles should rebound elastically. Here we simply plow past some of the problem's singularities with a "fill-in rule" of a non-differential equation type.

Oftentimes, as John Norton implicitly observes, popular recipes for patching over the descriptive holes in the vein of (ii) carry the risk of introducing a measure of certifiable indeterminacy in their wake. The classic example of this circumstance (in a CM context) can be found in the Riemann/Hugoniot recipe for repairing the shock wave blowups that otherwise commonly arise with respect to the natural equations for a confined gas. This repair recommends that what are now called "weak solutions" should be tolerated for these equations, but, without 
some further constraints, this new allowance tolerates far too many new solutions of this generalized type, with an attendant loss of solution uniqueness. To be sure, by evoking the celebrated Lax conditions as additional rules of thumb, we can restore unique weak solution developments within many one-dimensional applications, although it is dubious that this repair will prove entirely adequate in three dimensions as well (resolving these matters has proved enormously difficult ${ }^{15}$ ). Generically, there are good reasons to doubt whether blow-up problems of this ilk can be completely repaired by such adjoined "rules of thumb," simply because such principles rarely track the stage-by-stage evolution of the system in the tight manner provided by a set of differential equations. Once again, the central phenomenon to which we should pay the greatest attention is the appearance of the "missing physics" descriptive holes engendered by the blowups: the apparent failures of determinism enter largely as an artifact of the fact that the "rules of thumb" commonly cited in repair are often unable to plug the descriptive holes thoroughly.

In any case, from a strict MP point of view that demands power law forces, Norton's hillside display of "indeterminism" shouldn't seem troubling at all, for advocates of these foundations shouldn't accept Norton's proffered equation of motion as an acceptable "slow variable" decomposition for the situation at hand. Indeed, the complications which David Malament ${ }^{16}$ has discussed indicate that, in any proper point particle modeling of the conditions near the top of the track, the real motions are likely to prove so elaborate that no "fast variable"/"slow variable" decomposition will prove admissible in that region at all. Accordingly, a stout MP advocate can properly retort, "Norton appeals to a perfectly rigid track in setting up his problem, but no such constraint can be properly justified as an approximation within this setting, for the great complexities of how the ball particles will interact with the track particles have been improperly idealized away in the unwarranted appeal to a 'rigid track'." Indeed, I augur that this MP response accurately captures the background thinking that explains why few working physicists are likely to be swayed in their conviction that "classical mechanics is deterministic" by Norton's example. ${ }^{17}$

Observe that the main factor that these complications in our discussion trace to the factor that puzzled Richard Montague: "Why have practicing classical physicists been so reluctant to complete the MP picture by embracing power law supplements or other expedients that would turn 'determinism' into a provable condition of the physics?" The proper rationale traces simply to the brute fact that, when we begin inspecting smallish things at close range, Mother Nature stops 
supplying any firm indication of any appropriate MP rules at all, but steers us in the direction of quantum physics instead. As all molecular modelers know, their sixth and twelfth power rules possess a first order experimental accuracy at best and that, insofar as one can successfully model close interactions in a "classical" vein at all, one must seat the sources of intermolecular attraction and repulsion upon some kind of extended blobs (some of the relevant experimental considerations to this end had become well known by the mid-nineteenth century). Because Mother Nature refuses to supply suitable guidance with respect to possible short range laws for point particles, practitioners often dismiss Montague's request to "fill in the missing physics" in MP by observing, "Oh, extended blobs are actually important at that scale size, not true point particles." In much this same "mass points are not our real concern" spirit, Gallavotti appeals to the extended size of planets when he dismisses the "physical relevance" of the collision singularities appearing in MP physics. ${ }^{18}$ When physicists offhandedly offer such excuses, we tacitly witness the unstable stool of "classical mechanics" slowly settling upon other foundational legs: if "extended blobs" become tolerated as our "fundamental classical entities," we must shift the parameters of our discussion of determinism into the realms of either PC or true CM. As we'll see, different sets of considerations need to be canvassed with respect to "determinism" in these arenas than seem pertinent when we operate in MP mode.

And we should recognize a fundamental divergence in methodological ambition that renders these issues particularly awkward in real life practice. Although from a modeling point of view, we should object to the appearance of singularities where some density or velocity blows up to infinity; from a mathematical point of view, we often greatly value these very same breakdowns, for, as Riemann and Cauchy demonstrated long ago, the singularities of a problem commonly represent the precise features of the mathemtical landscape we should seek in our efforts to understand how the qualitative mathematics of a set of equations behaves. Inosfar as the project of achieving mathematical understanding goes, singularities prove our best friends, not our enemies. Accordingly, if we have already decided that our MP formulations overlook the missing physics pertinent to extended bodies anyway, why should we wish to junk up the mathematical formalism of MP with artificial assumptions concerning repulsion at close quarters? After all, such supplements merely camouflage the very singularities that we will want to uncover in understanding how our system behaves when no danger of close contact looms. Once we decide that MP shouldn't be considered to be a suitable formalism for modeling essential varieties 
of expected "classical process," we might as well keep the singularities that naturally arise within its bounds as nakedly transparent from a mathematical point of view as possible (taming the singularities only impedes understanding; it doesn't improve modeling accuracy). In other words, once we decide that the point masses of MP cannot adequately serve as "foundational entities" for classical physics considered as a whole, we simultaneously lose any motive for regarding MP's sundry breakdowns as deficiencies. Plainly, such methodological considerations will shape textbook presentations of MP doctrine along considerably different axes than our naïve "How does classical mechanics describe the world?" expectations anticipate. And this background explains why the strange "gaps" that Richard Montague noted within conventional formalisations of MP physics aren't really so surprising after all.

Once we foundationally substitute extended objects for point masses, we have shifted to a different framework (PC or CM) where the question of determinism can easily require a considerably different discussion. In fact, Norton's example nicely indicates how this can happen, for its particulars suits the expectations native to the physics of perfect constraints (PC), where extended objects ${ }^{19}$ are allowed to frame perfectly rigid surfaces upon which a suitable finite $\mathrm{set}^{20}$ of generalized coordinates can be installed and with respect to which the principle of generalized inertia proves completely valid. However, a number of fresh subtleties immediately intervene, the first of which is that "analytical mechanics" is rarely presented in a manner where its intended scope is clearly specified. The most common frameworks for implementing this flavor of physics utilize either a Lagrangian or Hamiltonian operator upon generalized coordinates in the context of holonomic constraints (although, as we shall soon observe, this last requirement is strangely limiting). The basic trouble is that most presentations are vague as to what should be tolerated under the heading of a "generalized coordinate" and this ambiguity makes it hard to evaluate the precise pertinence of Norton's example to PC in turn. For example, consider a ball freely sliding along a tube with a sharp bend and let the quality $\mathbf{S}(\mathbf{t})$ mark its increasing arc length displacements along its path. Does this ball and tube system fall within the proper ambit of PC? That answer depends upon whether $\mathbf{S}(\mathbf{t})$ qualifies as an acceptable "generalized coordinate" or not. But this issue looks as if it can be reasonably resolved in three ways. Answer 1: no, because $\mathbf{S}(\mathbf{t})$ doesn't possess completely smooth derivatives with respect to regular Cartesian

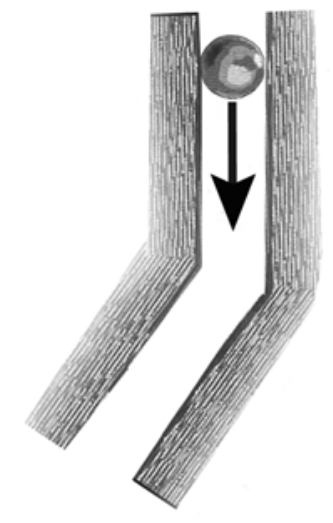


coordinates. Answer 2: yes, because the ball's generalized inertial motion proves perfectly smooth relative to $\mathbf{S}(\mathbf{t})$ itself (I'll supply answer 3 in a minute). However, if we accept this second answer, shouldn't we also allow a tube and ball system that splits both to the left and right as an acceptable PC system as well, for $\mathbf{S}(\mathbf{t})$ still appears as if it can serve as a suitable generalized coordinate for a ball rolling along a bifurcated tube? Granting $\mathbf{S}(\mathbf{t})$ this status, our split tube setup looks to be prima facie indeterministic under the assumption that generalized inertia must carry our ball along one tube or other past the forking. In fact, I have seen precisely this case, with these same implicit assumptions, cited to prove that "classical mechanics is indeterministic." 21 However, we can reasonably reject this possibility if we either retreat to answer 1 or (this is the delayed "answer 3") we simply declare $\mathbf{S ( t )}$ unacceptable as a "generalized coordinate" in this case simply because no complete set of admissible coordinates ${ }^{22}$ manages to fix the ball's state uniquely! In other words, answer 3 renders PC deterministic by fiat: any appeal to "generalized coordinates" for a system already presupposes that the system enjoys a set of coordinates that certify its motions as deterministic. In truth, split tube arrangements are usually not tolerated as "part of analytical mechanics" simply because no one really wants to bother with such outré states of affair when systems are studied from this point of view. To those who fancy that PC aspires to embrace "all of classical mechanics," this exclusion should seem arbitrary, but, in fact, "analytical mechanics" has no business pretending that it can successfully accommodate every intuitively expected "classical situation" anyway (its credentials for this foundational office are much worse, I think, than even those for MP). But once its inherent descriptive gaps are cheerfully acknowledged, applied mathematicians can reasonably demand that mathematical understanding should trump complete physical modeling when they carve out a proper arena for "analytical mechanics," for reasons similar to those that we canvassed in point mass circumstances. From this point of view, answer 3 isn't unreasonable at all.

Incidentally, if we adopt answer 1's approach to "generalized coordinates" and add a few supplementary assumptions, ${ }^{23}$ we might save determinism in this case by claiming that the unique "correct resolution" to our divided tube problem is one where the ball rebounds up the tube in a reversed direction (indeed, I have often heard this response offered as a "solution" to the indeterminism problem). However, Norton's example nicely demonstrates that this kind of answer is not 
sufficiently general--the singularity at the tube's bifurcation point can be sufficiently smoothed to make the non-unique continuations appear "more normal." To see how this can happen, let us consider another example which is often cited to demonstrate "classical non-determinism" in the older literature. Suppose we have a locomotive wheel and rod in the configuration sketched, where some large motive thrust $\mathbf{F}$ is applied along the piston rod (engineers call such configurations the "dead points" of the mechanism; their presence creates great headaches in real life design work). In which direction will the wheel turn under F's influence? As matters presently stand, $\mathbf{F}$ is perfectly matched by the "forces of constraint" that arise within

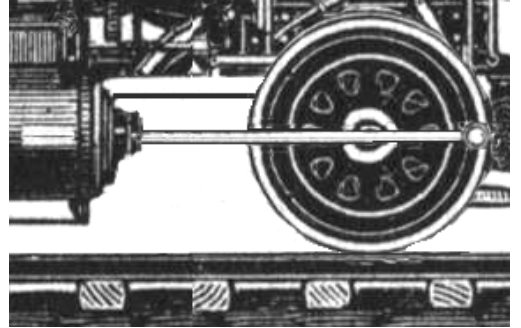

Dead point configuration the wheel, so the summed applied force "performs no work" on the wheel. However--and here is where the underlying parallels with Norton's hillside case become palpable--, as soon as the piston becomes slightly inclined from the horizontal, the altered geometry will allow some of the thrust $\mathbf{F}$ to "do work" on the wheel and accelerate its turning motion. Sometimes it is objected that "Really the wheel can never move from its 'dead spot' configuration because its acceleration must display an unacceptable jump to do so," in the same vein as we "solved" the tube indeterminacy. However, if our wheel happens to be shaped like the top of Norton's dome, then such "constrained force converting to active force" scenarios can be rendered smooth enough to bypass this standard objections. In any case, it's hard to remain consistently prissy about smooth movements within the realm of mechanism, which frequently display impulsive reversals in all sorts of natural context.

Such concerns represent but the tiny tip of a much larger iceberg of woes that lurk within the PC universe. As soon as we admit rigid objects of a finite geometry into "classical physics," it comes hard to accommodate the full range of expected set up variations within the framework of any fixed formalism. We have just witnessed a basic prototype: if God can build a tube with a sharp bend to the left, why can't He also build one that splits in both directions? "Because it doesn't suit my formalism" seems like a shabby answer. Nor are we likely to look favorably upon physicists who claim that balls can't roll and skaters can't glide down hillsides because such motions aren't tolerated within their favored form of PC. "Surely it's much easier to build a ball or a skate than anything that will 'purely slide' in your fashion," we complain. "Surely you've omitted much of the basic physics that governs classical contact interaction from your formalism." 
Although careless readers often overlook the fact, the familiar forms of "analytical mechanics" found in most textbooks tacitly demand "holonomic constraints" which can accommodate neither rolling nor skating. And even after these significant lapses in coverage are corrected by adopting a "virtual work" framework for PC better suited to rolling and sliding, the revised formalism will still prove unable to handle situations of over-constraint as exemplified within a modification of Aristotle's celebrated double-axled wheel, where the two hubs are mounted on rails in rack-and-pinion fashion. PC lacks any method for resolving how such a device will resolve the incompatible constraints restricting its free motion. ${ }^{24}$ Our immediate intuitive reaction to this over-constraint is to note that the interior of the wheel must somehow flex in response to these applied stresses: "Your analytical mechanics has left out the physics of all that," we complain. Quite right, but to tolerate flexure is ipso facto to depart the happy land of perfect constraints (PC) and instead take up foundational residence within the

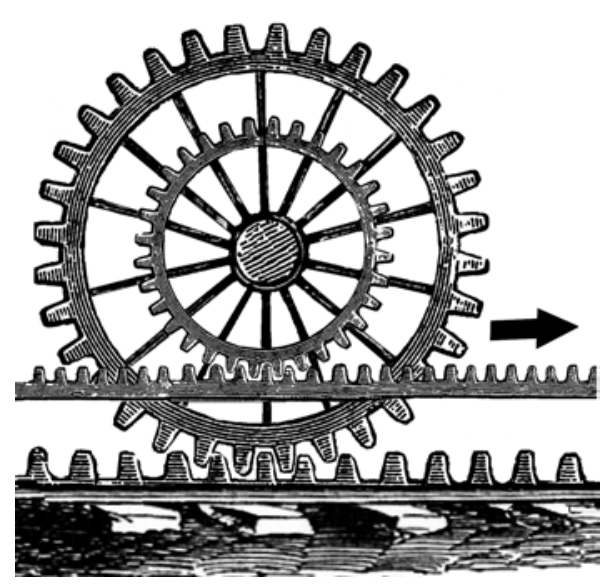

Wheel in over-constraint realm of pure continua $(\mathrm{CM})$ where perfectly rigid bodies are normally rejected as impossible, strictly speaking. Once again the specter of "missing physics" has caused the stool of "classical mechanics" to rock over to new foundational legs.

Before we briefly inspect the CM situation, let me comment upon a peculiar feature of classical analytical mechanics' present centrality within physics. Although rightly understood, it is hard to regard the formalism of analytical mechanics as capturing "the full world of classical mechanics" in any acceptable way, it nonetheless serves as an important guide, through standard quantization procedures, as to how the laws of the quantum world behave. For whatever reason, the symplectic structures et al. natural only to gliding but not rolling balls seem just the ticket to success with respect to quantum foundations. But we should not let this strange "success in guiding quantization" trick us into overlooking the descriptive gaps that analytic mechanics tolerates within its originally intended classical home.

Turning now to CM, we find that we can scarcely evade the problems caused by finite geometries, although they now arise in more subtle ways.. "If God can cut a rounded notch into a steel rod, why can't He cut an entirely sharp notch as well?," we wonder. Yet orthodox models for a sharply notched rod demand a 
blowup singularity in the stress at the notch. Realistically, we know that steel will flow plastically or even fragment long before any extremely high stress is achieved, but our formalism has not demanded that the physics needed to activate these processes be installed within our steel. Should we require it to do this? Well, materials scientists have developed some fairly good stories for plastic flow

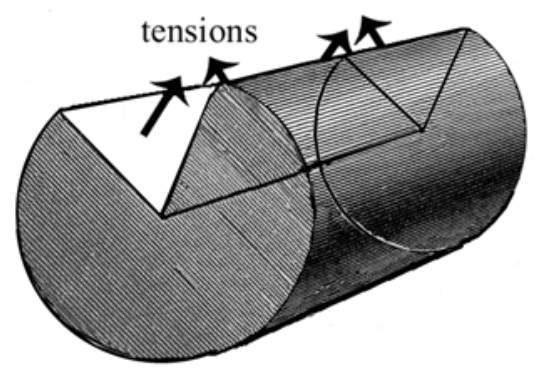
along this line, but they don't regard any of them as better than first order approximations. So we again face an uncomfortable dilemma much like our reluctance to embrace wholeheartedly the power law repulsion rules utilized by the molecular modelers; we can tame our notch anomaly through a "missing physics" rule of thumb that we don't particularly trust. And even if we do this, certain natural geometries are apt to introduce singularities within these revised models as well. In the meantime, the mathematicians continue to advise us, "Wait a minute! We like those singularities. Don't smooth them over with some crude rule of thumb that you don't really trust anyway." Accordingly, workers in continuum mechanics have instead attempted to discover pleasing but rather ad hoc compromises between restrictions upon the range of set up geometries they tolerate and answers that accept certain singularities within some specified flavor of generalized "solution". Efforts along these lines have forced the applied mathematician to consider very delicate flavors of function spaces, often adapted to different sorts of problem in different ways. So what do "classical mechanics' foundational objects" look like within continuum mechanics in light of these methodological subtleties? It becomes very hard to say. We might look to Mother Nature for foundational guidance, but, when pressed about classical physics, she only smiles disagreeably and points to quantum chemistry as the correct story of what occurs at such scale lengths.

Accordingly, despite our philosophical wishes otherwise, it seems unlikely that we'll ever manage to get our classical stool to sit firmly at rest on solid legs, simply because some flavor of "missing physics" consideration seems always ready to rock us off our present perch. We simply don't know how to fill out "the world of classical physics" in any consistent manner that doesn't tolerate strange gaps where some otherwise expected "classical situation" becomes disallowed on seemingly arbitrary grounds (indeed, often the circumstances that stymie classical foundational story $\mathrm{F}$ can be nicely modeled within the framework of some alternative classical story $\mathrm{G}$ and vice versa--a foundational instability that I have 
elsewhere called "the lousy encyclopedia phenomenon" 25 ). As long as such gaps persist, Norton-like indeterminancies may sometimes creep in, largely as a consequence of having adopted some fill-in "rule of thumb" (e.g., the constraint provided by Norton's perfectly rigid track) which we don't believe truly "gets all of the classical physics of the real life situation right." Accusations of "indeterminism" rarely seem definitive in such cases, simply because we've never really trusted the "rules of thumb" upon which they trade in the first case. This is why "missing physics" gaps of the sorts we have surveyed represent a more central feature of classical mechanics' peculiar circumstances than any of its potential indeterminacies. And these basic foundational considerations, it seems to me, supply the true explanation of why long ago Richard Montague wasn't able to locate enough ingredients to settle rigorously whether "classical mechanics" is deterministic or not. 


\section{Notes:}

1. This essay was written for a 2006 U.C.L.A. symposium on determinism. I would like to thank the other participants (especially David Malament, John Norton and Sheldon Smith) for helpful comments.

2. "The Dome: An Unexpectedly Simple Failure of Determinism," Phil Sci Arch (2006) and Philosophy of Science Assoc. 20th Biennial Mtg (Vancouver, BC), 2006. Norton calls his supporting structure a "dome," but some unstated constraint allows us to ignore the ball's potential angular displacements, producing the net effect of a track-like constraint. Hence I shall describe the situation as a "hillside track" in this paper.

3. Wandering Significance (Oxford: Oxford University Press, 2006).

4. As such, it represents the simplest prototype for how analytical mechanics diverges from point mass mechanics. By the way, I write "swooshing" because the resulting motion is neither a true sliding or rolling, which can engender surprisingly different behaviors on a two-deimnsional hillside.

7. To articulate the situation more accurately, the gravitational force can convey the particle downward, but the repulsive forces arising from the dome's surface must be smart enough to halt its descent in the right places with the correct tangential velocities.

6. In his odd Principles of Mechanics, Heinrich Hertz struggled to resolve our dilemma by nominating the constraint forces as primary while relegating the "active forces" to the humbler status of "artifacts of an approximation policy."

7. Giovanni Gallavotti, The Elements of Mechanics (New York: Springer-Verlag, 1983), p. 155. He decides to tolerate velocity dependent forces in his initial postulates, specializing to forces derived from a potential only later. Also: p. 169.

8. In real life, ragged surfaces lubricated by an intervening fluid are required before any simulacrum of frictionless sliding can be achieved (otherwise the ball binds tightly to the dome). The "constraints" favored in analytic mechanics model the contact between solids in a quite unrealistic manner whereas continuum mechanics contains better resources for this purpose. 
9. In practice, such modelers often evoke square well potentials as an expedient, but this coarse rule of thumb isn't compatible with the smoothness presumed in Newton's Second Law.

10. Richard Montague, Formal Philosophy (New Haven: Yale University Press, 1974).

11. Clifford Truesdell, "Suppesian Stews" in An Idiot's Fugitive Essays on Science (New York: Springer, 1984).

12. Donald Saari, Collisions, Rings and Other Newtonian N-body Problems (Providence: AMS, 2005).

13. John Earman, Determinism: A Primer, (Dordrecht: Kluwer, 1986).

14. Specifically, Earman and Norton argue that we should examine MP mechanics in a manner that tolerates hypothetical "space invaders" allowed to enter a regular MP scenario from spatial infinity in finite time. If so, this tolerance should be described in terms of a novel "side condition at infinity" added to conventional MP rather than an "initial condition" per se (following Hadamard, mathematicians have performed a very valuable service in classifying the sundry kinds of natural "side condition" pertinent to differential equations precisely and I believe we should not trample upon their good terminological offices unduly). In view of its rather extensive foundational holes, I am unpersuaded that such novel "side conditions" represent the best way to highlight what is troublesome in orthodox MP physics.

15. V.I. Yudovich, "Global Solvability Versus Collapse in the Dynamics of an Incompressible Fluid" in A.A. Bolibruch, Yu. S. Osipov and Ya. G. Sinai, Mathematical Events of the Twentieth Century (Berlin: Springer, 2006).

16. David Malament, "Norton's Slippery Slope”, Pittsburgh Phil Sci Arch (2007) and Philosophy of Science Assoc. 20th Biennial Mtg (Vancouver, BC), 2006.

17. Indeed, V.I. Arnold in his celebrated Mathematical Methods of Classical Mechanics (Berlin: Springer, 1997) assumes determinism as an axiom!

18. Gallavotti, op cit., p. 30.

19. A related subtlety merits a passing remark, even if it does not touch upon determinism per se. Strictly speaking, Norton's example involves a dimensional 
mismatch between an extended and an unextended object: a zero dimensional point situated upon the two dimensional surface of a hillside. It is common practice to study examples of this sort, but should we really wish to accept such mismatches as foundationally basic? Concrete experience with continua suggests otherwise. The history of the subject is full of treatments where a three dimensional system such as a plank has been mathematically treated as a two-dimensional or one dimensional array, often by appeal to some apparent symmetry in its configuration. Nonetheless, it is now recognized that these reduced treatments are hard to justify rigorously and that it is a mistake to apply fundamental mechanical axioms to such lowered dimensional systems directly. With respect to the dome case, three dimensional balls often act quite unexpectedly on frictionless planes and one should be careful about presuming that Norton's predicted motion will emerge in any reasonable limit as a three dimensional ball is reduced in size towards a point (this is why I called Norton's predicted motion a "swooshing" rather than a rolling or sliding). However, this quibble, although important for foundational work, does not affect Norton's example in any material way, for we can reproduce analogs of his circumstances in higher dimensions.

20. Historically, the great interest in Lagrangian mechanics and other forms of variational principle lay in the hope that detailed hypotheses about local contact action could be evaded by such means--see Olivier Darrigol, Worlds of Flow (Oxford: Oxford, 2005), ch. 1. The intended arena is continuum mechanics, with finite dimensional modelings emerging only as first order approximations through "lumping." The notion that finite dimensional "analytical mechanics" might prove foundationally central emerged only later, with no clear parent insofar as I am aware. But Norton's example is troubling only if we adopt such a point of view.

21. Clifford A. Truesdell, Six Lectures on Modern Natural Philosophy (Berlin: Springer-Verlag, 1966).

22. To have a complete set of coordinates for the split tube case, we also need a variable to mark lateral position within the tubes and this variable won't normally prove smooth (although we can improve matters by tricks like Norton's).

23. In accepting the rebounding state of affairs as a "solution," we tacitly tolerate a trajectory that falls outside the usual requirements of analytic mechanics. An adequate investigation of "determinism" relevant to such circumstances will require a more precise delineation of the range of "weak solutions" tolerated. 
24. Hertz' system of rigid body-based mechanics was often criticized for accommodating over-constraint of this sort.

25. Wandering Significance, p. 180. 